\title{
Evolución del índice de masa corporal en niñas con pubertad precoz en tratamiento
}

\author{
Change in body mass index among girls with precocious puberty under \\ treatment
}

\author{
Dra. Jessie N. Zurita Cruz ${ }^{b}$ Dra. Irene Díaz Rodrígueza, Dra. Elisa Nishimura Meguro, \\ Dr. Miguel Á. Villasis Keever ${ }^{b}$, Dra. Aleida De Jesús Rivera Hernández y Dra. Eulalia Garrido Magaña
}

\begin{abstract}
RESUMEN
Introducción: Las modificaciones del índice de masa corporal (IMC) en niñas con pubertad precoz central idiopática (PPCI) bajo tratamiento han sido controversiales en otras poblaciones. Objetivo: Evaluar la modificación de puntaje z de IMC y la presencia de sobrepeso y obesidad en niñas mexicanas con PPCI durante su primer año de tratamiento.

Metodología: Se realizó un estudio retrospectivo de niñas con PPCI, tratadas con leuprolide, con medición semestral de la somatometría por un año. Se utilizó análisis de la varianza (analysis of variance; ANOVA, por sus siglas en inglés) para las diferencias del puntaje $\mathrm{z}$ de IMC y análisis multivariado para la relación con los diferentes factores.

Resultados: En 121 niñas, el puntaje z de IMC se incrementó de 0,87 a 1,32, y la presencia de obesidad y sobrepeso aumentó de $40,5 \%$ a $70,3 \%$, con diferencia estadísticamente significativa. Conclusiones: La modificación de puntaje $\mathrm{z}$ de IMC y la frecuencia de sobrepeso $u$ obesidad en niñas mexicanas con PPCI aumentaron significativamente.

Palabras clave: pubertad precoz central, índice de masa corporal, obesidad, leuprolide.
\end{abstract}

http:/ /dx.doi.org/10.5546/aap.2016.143

\section{INTRODUCCIÓN}

La pubertad precoz central (PPC) se define como el desarrollo de las características sexuales secundarias antes de los 9 años de edad en los varones y de los 8 años en las niñas, por activación prematura del eje hipotálamo-hipófisis-gónada. ${ }^{1}$

\footnotetext{
a. Departamento de Endocrinología Pediátrica.

b. Unidad de Investigación Médica en Epidemiología Clínica. Unidad Médica de Alta Especialidad (UMAE), Hospital de Pediatría Centro Médico Nacional Siglo XXI.

Instituto Mexicano del Seguro Social.

México.
}

Correspondencia:

Dra. Jessie N. Zurita Cruz: zuritajn@hotmail.com

Financiamiento: Ninguno.

Conflicto de intereses: Ninguno que declarar.

Recibido: 8-9-2015

Aceptado: 11-11-2015

Publicado Primero en Internet 25-2-2016
El tratamiento de primera elección de la PPC es con análogos de acción prolongada de la hormona liberadora de gonadotrofinas (análogo de gonadotropin-releasing hormone; aGnRH, por sus siglas en inglés), con el cual se logra la supresión puberal en los primeros 3 meses de tratamiento en la mayoría de los casos. ${ }^{2,3}$ Los efectos adversos son poco frecuentes; entre ellos, se ha reportado el incremento desproporcionado del índice de masa corporal (IMC) ${ }^{4-8}$ a expensas del tejido graso, con el consecuente riesgo cardiovascular. El mecanismo por el cual estas pacientes tienen mayor predisposición al sobrepeso u obesidad es incierto y los hallazgos en las diferentes series son inconsistentes. ${ }^{9,10}$

En México, el sobrepeso en escolares y adolescentes es un problema de salud pública y refleja los hábitos de alimentación y actividad física en una población con mayor riesgo genético.

El objetivo de este estudio es evaluar la modificación de puntaje $\mathrm{z}$ de índice de masa corporal $(\Delta$ szIMC) y la presencia de sobrepeso y obesidad en niñas mexicanas con pubertad precoz central idiopática (PPCI) durante su primer año de tratamiento.

\section{PACIENTES, MATERIAL Y MÉTODOS}

Se realizó una cohorte retrospectiva de niñas mexicanas con diagnóstico de PPCI, bajo tratamiento ininterrumpido con leuprolide en dosis mensuales de 3,75 mg durante 12 meses. Se excluyeron pacientes con alguna enfermedad asociada o medicamentos que potencialmente influyeran en la ganancia ponderal o el incremento del apetito (síndromes genéticos, uso de esteroides, fluoxetina, sensibilizadores a insulina, hiporexigénicos o inhibidores de absorción intestinal grasa).

Del expediente clínico, se tomaron los datos clínicos, como edad, peso, talla, estadio puberal, edad ósea y determinaciones séricas de la hormona luteinizante (luteinizing hormone; LH, 
por sus siglas en inglés), foliculoestimulante (follicle stimulating hormone; FSH, por sus siglas en inglés) y estradiol, al momento del diagnóstico, 6 y 12 meses después. Se consideró sobrepeso un IMC con percentil (PC) de 85 a 94 y obesidad, un $P C$ igual a 95 o mayor.

El protocolo fue aprobado por parte del Comité Local de Investigación y Ética en Salud del Hospital correspondiente.

Mediante ANOVA de una vía, se evaluó la diferencia de puntaje $z$ de índice de masa corporal (szIMC) al momento del diagnóstico, 6 y 12 meses después. Se calculó la $\triangle$ szIMC al restar el szIMC a los 12 meses y al diagnosticar la PPCI. Estos datos se analizaron con el estado nutricional basal, la presencia de uno o ambos padres con obesidad y el grado de supresión bioquímica de la PPCI (SPSS versión 17.0).

TABLA 1. Características generales de las 121 pacientes con pubertad precoz central idiopática al momento del diagnóstico

\begin{tabular}{lcc}
\hline Característica & & Promedio (DE) \\
\hline Edad (años) & & $6,8(1,29)$ \\
Peso (kg) & & $32,5(10,5)$ \\
Talla (cm) & & $129,5(12,77)$ \\
szIMC & & $0,86(1,06)$ \\
Edad ósea (años) & $10,3(2,2)$ \\
\hline Estadio puberal \% (Tanner*) & 2 & 86,8 \\
& 3 & 13,2 \\
\hline Obesidad en los padres* & Ninguno & 6,61 \\
& Uno & 82,64 \\
& Ambos & 10,74 \\
\hline Perfil bioquímico & LH (UI/L) & $4,2(5,4)$ \\
al momento del & FSH (UI/L) & $6,5(8,5)$ \\
diagnóstico & Estradiol (pg/ml) & $38,6(3,2)$ \\
\hline
\end{tabular}

DE: desvío estándar; LH: hormona luteinizante; FSH: hormona foliculoestimulante;

szIMC: puntaje $\mathrm{z}$ de índice de masa corporal.

\section{RESULTADOS}

De un total de 147 pacientes atendidas por PCCI en el período de 2005 a 2012, se excluyeron 17 pacientes por tener diagnóstico de hiperplasia suprarrenal congénita, 6 por tumor del sistema nervioso central (4 astrocitomas y 2 hamartomas) y otras 3 pacientes porque no habían recibido el tratamiento de manera continua durante los 12 meses. Se incluyeron 121 pacientes, con edad promedio al momento del diagnóstico de 6,8 \pm 1,2 años. El 86,7\% $(n=105)$ se encontraba en estadio de Tanner 2 y el 13,2\% $(n=16$,$) , en estadio 3$. En el 95\% de los casos, la pubertad se mantuvo suprimida clínica y bioquímicamente (Tabla 1).

Además, en cuanto al estado de nutrición en el momento del diagnóstico, el promedio del szIMC fue de $0,86 \pm 1,06$, y se identificaron 60 pacientes $(49,5 \%)$ con estado de nutrición normal, 35 (29\%) con sobrepeso y 26 (21,5\%) con obesidad. Al agrupar estos últimos, en total, se identificaron 61 pacientes $(50,4 \%)$ con sobrepeso $\mathrm{u}$ obesidad. La $\Delta$ szIMC fue de $0,46 \pm 0,66 \mathrm{~kg} / \mathrm{m}^{2}$ $(\mathrm{p}=0,001)$, lo que se reflejó en un incremento en los porcentajes de sobrepeso y obesidad a 33\% y $37 \%$, respectivamente (Tabla 2, Figura 1).

El incremento significativo en el szIMC se observó, principalmente, en el grupo con peso normal o sobrepeso. La proporción de niñas que modificaron su condición nutricional a sobrepeso y obesidad a los 12 meses de tratamiento fue de $46,6 \%$ en el grupo con szIMC inicial < PC 85 y, en el grupo con sobrepeso, el 25\% evolucionó a obesidad (Figura 2).

Hubo 6 casos en los cuales no se logró la supresión bioquímica adecuada de la pubertad, principalmente, por irregularidad en la administración del aGnRH. En este subgrupo, el incremento en el szIMC fue de magnitud similar al grupo bien suprimido ( $\triangle$ szIMC: $0,74 \pm 0,65)$, aunque no se pudo demostrar significancia estadística con la condición basal, probablemente

TABLA 2. Estado nutricional durante el seguimiento de 121 pacientes con pubertad precoz central idiopática

\begin{tabular}{|c|c|c|c|c|}
\hline & & \multicolumn{3}{|c|}{ Promedio (DE) } \\
\hline & & Basal & 6 meses & 12 meses \\
\hline \multicolumn{2}{|c|}{ szIMC $^{*}$} & $0,86(1,06)$ & $1,1(0,97)$ & $1,3(0,88)$ \\
\hline \multirow[t]{3}{*}{ Estado de nutrición ${ }^{* *}$} & Normal & 49,5 & 37 & 29,7 \\
\hline & Sobrepeso & 29 & 33 & 33,1 \\
\hline & Obesidad & 21,5 & 30 & 37,2 \\
\hline
\end{tabular}

*ANOVA szIMC: basal vs. 6 meses, $p=0,096 ; 6$ vs. 12 meses, $p=0,395$; basal vs. 12 meses, $p=0,001$.

** Porcentaje.

DE: desvío estándar; szIMC: puntaje z de índice de masa corporal. 
por el número de casos. El análisis multivariado no demostró diferencia en la $\triangle$ szIMC de acuerdo con las concentraciones de LH o el antecedente de uno o ambos padres con obesidad.

\section{DISCUSIÓN}

Los problemas cardiovasculares y otras enfermedades asociadas a la obesidad son la principal causa de morbimortalidad en muchos países, entre los que se incluye México. En la edad pediátrica, el aumento en la proporción de grasa corporal se ha relacionado con la maduración biológica temprana e, incluso, la presencia de pubertad precoz.

Lo anterior podría reflejar la historia natural de estas niñas, ya que los factores que condicionan el sobrepeso u obesidad en estas pacientes continúan presentes (carga genética, sobrealimentación, sedentarismo). Además, la supresión puberal podría contribuir al desequilibrio ingesta-gasto calórico, al disminuir, teóricamente, el gasto atribuible al crecimiento longitudinal y desarrollo de caracteres sexuales secundarios. ${ }^{10}$

En este estudio retrospectivo, en el cual no se controló específicamente la actividad física o la alimentación, observamos que la mayoría de las niñas con sobrepeso $\mathrm{u}$ obesidad continuaron en esta condición y que el $40 \%$ de las niñas previamente eutróficas desarrollaron sobrepeso $\mathrm{u}$ obesidad. Los datos anteriores se resumen en $70 \%$ de sobrepeso u obesidad a los 12 meses de tratamiento vs. $50,4 \%$ al iniciar el tratamiento. La magnitud del incremento del szIMC (de 0,86 a 1,3; $\Delta$ szIMC: $0,46 \pm 0,66)$ es mayor que la reportada

Figura 1. Evolución del puntaje z de indice de masa corporal en niñas con pubertad precoz central idiopática

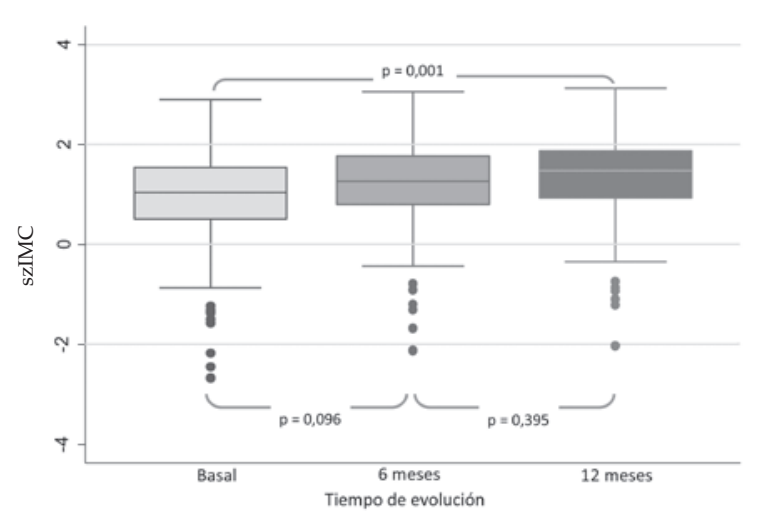

ANOVA de una vía, con análisis post hoc de Bonferroni. szIMC: puntaje z del índice de masa corporal por $B_{0 o t}^{4}$ (de 0,96 a 1,38; $\Delta$ szIMC: 0,42) en 34 niñas caucásicas después de 2 años de tratamiento y por Aguiar $^{7}$ en 176 niñas brasileñas tratadas con goserelina, aunque la proporción de casos con sobrepeso u obesidad es mayor que el $30 \%$ (vs. el 19\% al momento del diagnóstico) reportado en 96 niñas caucásicas por Palmert y col. ${ }^{5}$

Además, en una cohorte de 115 niñas israelitas ${ }^{8}$ tratadas con leuprolide durante 2-3 años, también se reporta una alta frecuencia de sobrepeso u obesidad, pero no se evidenciaron cambios en el szIMC. Incluso, hay un estudio de 101 niñas italianas en las que el szIMC disminuyó significativamente bajo tratamiento. ${ }^{9}$ En ninguno de los estudios referidos, se señala específicamente si se realizó alguna intervención preventiva. Este incremento del szIMC puede deberse a las características particulares de este grupo étnico, como una mayor predisposición genética a las alteraciones cardiometabólicas y factores medioambientales.

Se comparó la frecuencia de sobrepeso y obesidad de los casos estudiados tanto por grupo de edad cronológica (6,8 $\pm 1,29$ años) como edad biológica $(10,6 \pm 1,8$ años) con la frecuencia reportada de este problema en niñas mexicanas en la Encuesta Nacional de Salud 2012. ${ }^{11}$ Es evidente que, al igual que en otras poblaciones, la frecuencia de sobrepeso y obesidad en niñas con pubertad precoz central idiopática es mayor que la reportada para la población general tanto para edad cronológica como biológica (40,5\% vs. $24,7 \%$ y $38,2 \%$ vs. $40,5 \%$, respectivamente). El incremento del szIMC fue, incluso, mayor que

FIGURA 2. Modificaciones del estado nutricional durante 12 meses de seguimiento. $N=121$

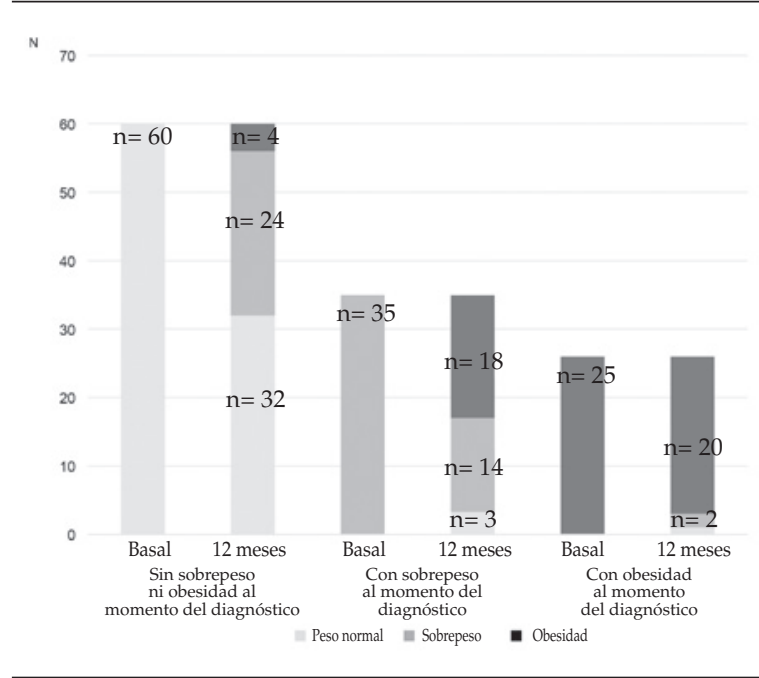


el reportado por el rebote de adiposidad normal entre los 5 y los 7 años de edad cronológica, ${ }^{12}$ que se caracteriza por un incremento aproximado del $16 \%$ de grasa total o 0,2 puntos en el szIMC.

$\mathrm{El}$ incremento desproporcionado de sobrepeso y obesidad, principalmente en las niñas con IMC normal al momento del diagnóstico, sugiere que, además de la herencia, sedentarismo y sobrealimentación, algún otro mecanismo podría contribuir a una mayor evolución hacia el sobrepeso u obesidad, por lo menos, en la población estudiada.

Dada la característica retrospectiva del estudio, no contamos con datos sobre otros factores de riesgo cardiovascular (lípidos, hiperinsulinemia, adipocitocinas), que se han reportado aumentados en niñas con pubertad precoz central bajo tratamiento que modifican su estado nutricional. ${ }^{13,14}$

En conclusión, el grupo de niñas mexicanas con PPCI a un año del diagnóstico presentó una mayor frecuencia de sobrepeso u obesidad que lo reportado en otras poblaciones. La tasa de progresión a obesidad también fue mayor, sobretodo en el grupo con peso normal al momento del diagnóstico, lo que sugiere que factores atribuibles a la supresión puberal pueden participar en el incremento ponderal. En niñas con pubertad precoz en poblaciones de alto riesgo de obesidad, como la nuestra, es importante implementar medidas de prevención, específicamente en la identificación y manejo de factores de riesgo, como parte del manejo integral de este padecimiento.

\section{REFERENCIAS}

1. Sorensen K, Mouritsen A, Aksglaede L, Hagen CP, et al. Recent secular trends in pubertal timing: implications for evaluation and diagnosis of precocious puberty. Horm Res Paediatr 2012;77(3):137-45.

2. Fuld $\mathrm{K}$, Chi $\mathrm{C}$, Neely EK. A randomized trial of 1- and 3-month depot leuprolide doses in the treatment of central precocious puberty. J Pediatr 2011;159(6):982-7.

3. Badaru A, Wilson DM, Bachrach LK, Fechner P, et al. Sequential comparisons of one-month and three-month depot leuprolide regimens in central precocious puberty.
J Clin Endocrinol Metab 2006;91(5):1862-7.

4. Boot AM, De Muinck Keizer-SchramaS, Pols HA, Krenning $\mathrm{EP}$, et al. Bone mineral density and body composition before and during treatment with gonadotropin-releasing hormone agonist in children with central precocious puberty and early puberty. J Clin Endocrinol Metab 1998;83(2):370-3.

5. Palmert MR, Mansfield MJ, Crowley WF Jr, Crigler JF Jr, et al. Is obesity an outcome of gonadotropin-releasing hormone agonist administration? Analysis of growth and body composition in 110 patients with central precocious puberty. J Clin Endocrinol Metab 1999;84(12):4480-8.

6. Pasquino AM, Pucarelli I, Accardo F, Demiraj V, et al. Long-term observation of 87 girls with idiopathic central precocious puberty treated with gonadotropin-releasing hormone analogs: impact on adult height, body mass index, bone mineral content, and reproductive function. J Clin Endocrinol Metab 2008;93(1):190-5.

7. Aguiar AL, Couto-Silva AC, Vicente EJ, Freitas IC, et al. Weight evolution in girls treated for idiopathic central precocious puberty with GnRH analogues. J Pediatr Endocrinol Metab 2006;19(11):1327-34.

8. Lazar L, Padoa A, Phillip M. Growth pattern and final height after cessation of gonadotropin-supressive therapy in girls in girls with central sexual precocity. J Clin Endocrinol Metab 2007;92(9):3483-9.

9. Arrigo T, De Luca F, Antoniazzi F, Galluzzi F, et al. Reduction of baselinebody massindexundergonadotropinsuppressive therapy in girls with idiopathic precocious puberty. Eur J Endocrinol 2004;150(4):533-7.

10. Sørensen K, Mourintsen A, Mogensen SS, Aksglaede L, et al. Insulin sensitivity and lipid profiles in girls with central precocious puberty before and during gonadal suppression. J Clin Endocrinol Metab 2010;95(8):3736-44.

11. Gutiérrez JP, Rivera-Dommarco J, Shamah-Levy T, Villalpando-Hernández S, et al. Encuesta Nacional de Salud y Nutrición 2012. Resultados nacionales. Cuernavaca: Instituto Nacional de Salud Pública de México; 2012:149-52. [Consulta: 28 de septiembre de 2014]. Disponible en: http://ensanut.insp.mx/informes/ ENSANUT2012ResultadosNacionales.pdf.

12. Williams S, Davie G, Lam F. Predicting BMI in young adults from childhood data using two approaches to modelling adiposity rebound. Int J Obes Relat Metab Disord 1999;23(4):348-54.

13. Mul D, Fredriks AM, van Buuren S, Oostdijk W, et al. Pubertal development in The Netherlands 1965-1997. Pediatr Res 2001;50(4):479-86.

14. GlueckCJ,MorrisonJA, Wang P. Insulin resistance, obesity, hypofibrinolysis, hyperandrogenism, and coronary heart disease risk factors in 25 pre-perimenarchal girls age $<$ or $=14$ years, 13 with precocious puberty, 23 with a firstdegree relative with polycystic ovary syndrome. J Pediatr Endocrinol Metab 2008;21(10):973-83.

\section{CORRECCIÓN}

Los autores del artículo original: "Impacto de la vacuna neumocócica conjugada 13-valente en la incidencia de neumonía consolidante en menores de 5 años en el partido de Pilar, Buenos Aires: estudio de base poblacional", publicado en Arch Argent Pediatr 2015;113 (6):502-9 solicitan que se aclare que los centros participantes han sido exclusivamente hospitales públicos del área de Pilar, los hospitales Hospital Pediátrico Falcón y Hospital Juan Sanguinetti. Por error se incluyó el Hospital Universitario Austral y sus profesionales no participaron en la elaboración del estudio ni contribuyeron con pacientes. 